\title{
Efficient Co-design of Composite Smart Structures (Antennas and Mechanical Structures) Using a Novel Hybrid Optimization Technique
}

\author{
C. You*(1), D. Staiculescu ${ }^{(1)}$, L. Martin ${ }^{(2)}$, W. Hwang ${ }^{(3)}$ and M. M. Tentzeris ${ }^{(1)}$ \\ (1) Georgia Institute of Technology, Atlanta, GA, 30332, U.S.A \\ (2) Motorola, Plantation, FL, 33322, U.S.A. \\ (3) Pohang University of Science and Technology, Pohang, 790-784, South Korea
}

\section{Introduction}

In the recent years, the need for improved structural efficiency and mechanical/electrical performance for the antennas, especially for aircraft applications, has led to their embedding of the functions and components in load-bearing structural surfaces for aircraft applications [1-3]. The use of a composite roof structure in military vehicles with multiple antennas ensures high electrical performance in a light-weight and durable self-contained structure [4]. Through the innovative integration of antenna elements, amplifiers and ground plane, the reception quality and manufacturability of vehicle-mounted modules is expected to be significantly improved. The present work aims to integrate the antenna with the mechanical structure, therefore co-design electrically and structurally effective antenna structures, named composite smart structures (CSS). Design is focused electrically toward high gain and wide bandwidth, and mechanically toward high structural strength and low density (low-weight). For the antenna function microstrip antennas are commonly used because they are conformable to planar and nonplanar surfaces, while sandwich structures composed of composite faces and honeycomb core are used as the basic supporting mechanical structure due to their high strength and low density. The integration procedure improves the gain of the antenna without affecting the bandwidth [5]. The electrical and mechanical analyses are incorporated into the Design of Experiments (DOE) and Response Surface Method (RSM) statistical optimization techniques, which give a thorough understanding of the system and, most importantly, give information such as how the electrical and mechanical performances are affected by each other, namely how they interact. Previous work [6] has demonstrated the successful use of hybrid statistical techniques in microwave system electromagnetic analysis and optimization, but this is the first reported work on incorporating the mechanical performance. The system is optimized with respect to all these factors simultaneously, also giving the designer the flexibility to choose the goals and the weights of each of the optimized outputs.

\section{Fundamental CSS Structures}

There are two different composite smart structures incorporating two different microstrip antenna configurations to be analyzed and optimized: $\mathrm{CSS}_{1}$, including a basic microstrip antenna, and $\mathrm{CSS}_{2}$ in which a stacked-patch antenna is inserted for increased bandwidth. Both configurations are presented in Fig. 1. The fundamental idea of the CSS panel is inserting the antennas in a composite sandwich. The sandwich construction involves two relatively dense and stiff facesheets that are bonded to either side of a lowdensity core. Compared to single material, the sandwich concept provides substantial improvements in both flexural rigidity and flexural strength. Glass/Epoxy composite materials are used as a facesheet material, and Nomex honeycombs as a core material. The Nomex honeycomb is made of Aramid fiber paper, formed in a honeycomb 
configuration and dipped in heat resistant resin. The cell size is $1 / 8$ ", and the density is 5 $\mathrm{lbs} / \mathrm{ft}^{3}$.

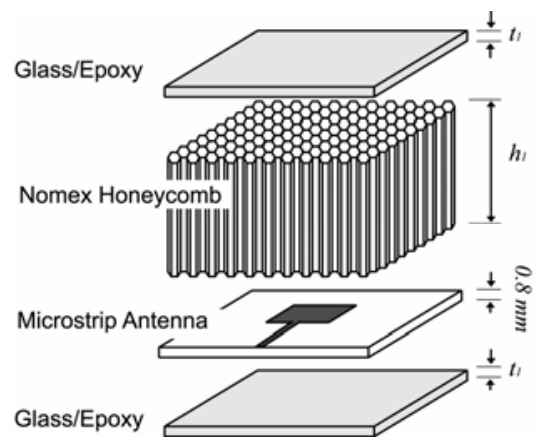

(a) $\mathrm{CSS}_{1}$

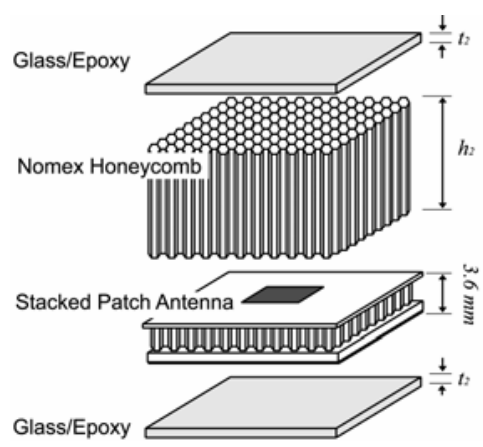

(b) $\mathrm{CSS}_{2}$

Fig. 1. Basic model.

First, the antennas performance have been modeled with IE3D (v. 10.06) and the resonant frequency is $12.2 \mathrm{GHz}$. The two parameters under investigation for the hybrid optimization, that covers both the electrical and mechanical performance of the system, are the thickness of the Nomex honeycomb, $h_{\mathrm{n}}$, and the thickness of the facesheet on the top of it, $t_{\mathrm{n}}$. The responses for the statistical models are the antenna gain $G_{\mathrm{n}}$ at the resonant frequency, flexural rigidity $D_{\mathrm{n}}$, and density $W_{\mathrm{n}}$. The purpose of the sandwich panels is to improve the mechanical resistance at bending and buckling without adding weight to the structure. Both the bending and buckling resistance depend on the flexural rigidity $D_{\mathrm{n}}$, which is the force couple required to bend the structure to a unit curvature [7]. $W_{\mathrm{n}}$ is the density of the entire structure.

The design space for the two parameters has been chosen such that it represents physically realizable values and incorporates fabrication limitations and design rules. Also, the range was chosen small enough to ensure both little change of impedance characteristic and high gain. In $\mathrm{CSS}_{1}$ it is found that the integration decreases the resonant frequency and the shift in the resonant frequency increases with the gain. Therefore, the ranges for the two input variables in $\mathrm{CSS}_{1}$ are selected for high gain condition with a reasonable core/face thickness ratio, that is, $1.5-2.5 \mathrm{~mm}$ for $t_{1}$ and 11.5 $12.5 \mathrm{~mm}$ for $h_{1}$. For $\mathrm{CSS}_{2}$, the integration increases the input port return loss and the return loss change increases with the gain also. Therefore, the ranges for the two input variables in $\mathrm{CSS}_{2}$ are selected for wideband performance, with a reasonable core/face thickness ratio, that is, $1-1.5 \mathrm{~mm}$ for $t_{2}$ and $8.5-9.5 \mathrm{~mm}$ for $h_{2}$.

\section{Statistical Analysis and Optimization}

The electrical and mechanical analyses are incorporated into the Design of Experiments (DOE) and Response Surface Method (RSM) statistical optimization techniques. The experimentation method chosen for the DOE is a full factorial design with center points. The factorial designs are used in experiments involving several factors where the goal is the study of the joint effects of the factors on a response. The design used was a $2^{k}$ factorial design with center points to increase capability of investigating model fit, including curvature in the response. The statistical analysis of the first order model shows which parameters are significant for each of the two figures of merit and the ones that are not significant are eliminated from further analysis. Then, the model is 
checked for curvature. If curvature in the response is detected, RSM can account for curvature through second-order model development. Usually, these second-order models are reasonable approximations of the true functional relationship over relatively small regions. Once validated, the models are approximately equivalent to the actual system within the defined design space [8].

In our case, the DOE analysis revealed curvature, and the analysis of the second-order RSM data showed the statistical significance of the inputs for the three figures of merit. For the gain $G_{\mathrm{n}}$, the significant terms are $t_{\mathrm{n}}, h_{\mathrm{n}}$, the interaction $t_{\mathrm{n}} \times h_{\mathrm{n}}$, and the second order terms $t_{\mathrm{n}} \times t_{\mathrm{n}}$ and $h_{\mathrm{n}} \times h_{\mathrm{n}}$ alleviated the curvature in the model. For the flexural rigidity $D_{\mathrm{n}}$ the significant terms are $t_{\mathrm{n}}$ and $h_{\mathrm{n}}$ and and $t_{\mathrm{n}}$ is the only term significant for the density $W_{\mathrm{n}}$. Before proceeding to the actual optimization, the models had to be confirmed. The confirmation of the models was performed for a combination of the input variables, first simulated in the electromagnetic simulator and also predicted with the developed models. The results of the simulation fell into the RSM 95\% confidence intervals defined by the lower and upper bounds for the predicted outputs, so the models have been confirmed.

The final step in our study is the actual optimization of the model. The optimization goals are to simultaneously maximize gain $G_{\mathrm{n}}$ (weight 0.4 ) and flexural rigidity $D_{\mathrm{n}}$ (weight 0.3 ), and minimize density $W_{\mathrm{n}}$ (weight 0.3 ). The surfaces for the three figures of merit as a function of the optimizing parameters are presented in Fig. 2. The optimization is done based on the plot in Fig. 3. The optimal was obtained for $t_{1}=2.1 \mathrm{~mm}$ and $h_{1}=$ $11.74 \mathrm{~mm}$, leading to the optimized values of $G_{1}=11.3 \mathrm{dBi}, D_{1}=5.78 \times 10^{6} \mathrm{Nmm}$ and $W_{2}$ $=10.23 \mathrm{~kg} / \mathrm{m}^{2}$ for $\mathrm{CSS}_{1}$, and $t_{2}=1.4 \mathrm{~mm}$ and $h_{2}=8.8 \mathrm{~mm}$, leading to the values of $G_{2}=$ $11.73 \mathrm{dBi}, D_{2}=3.4 \times 10^{6} \mathrm{Nmm}$ and $W_{2}=8.25 \mathrm{~kg} / \mathrm{m}^{2}$ for $\mathrm{CSS}_{2}$. The RSM optimized structure was simulated and the values obtained for the output variables agreed well with those predicted by the statistical models.
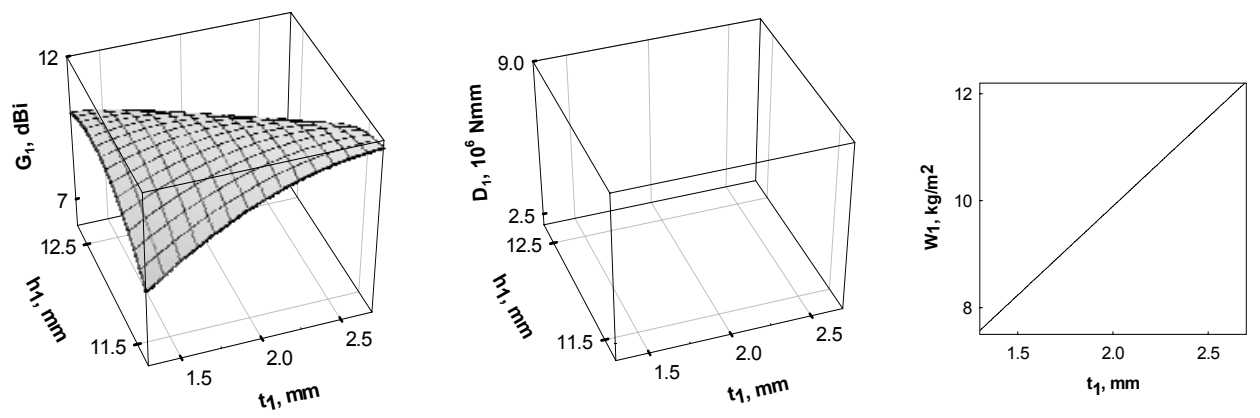

(a) $\mathrm{CSS}_{1}$
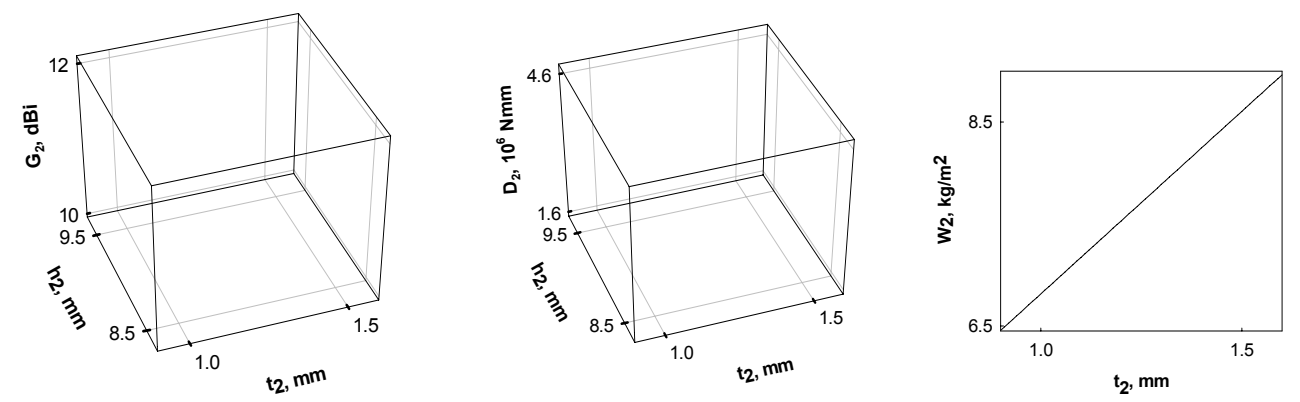

(b) $\mathrm{CSS}_{2}$ 
Fig. 2 Surfaces of possible solutions for optimized $G_{\mathrm{n}}, D_{\mathrm{n}}$ and $W_{\mathrm{n}}$

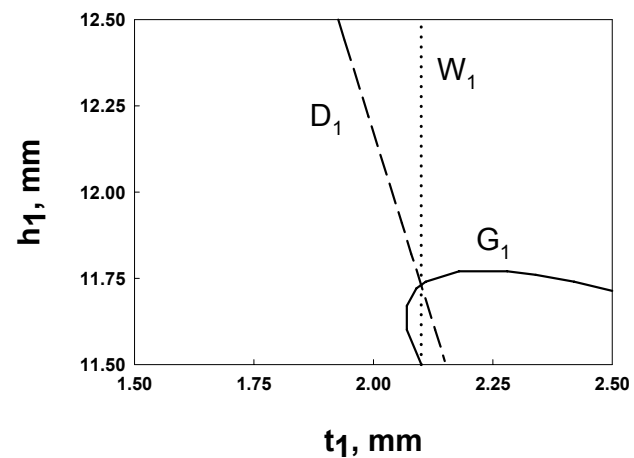

(a) $\mathrm{CSS}_{1}$

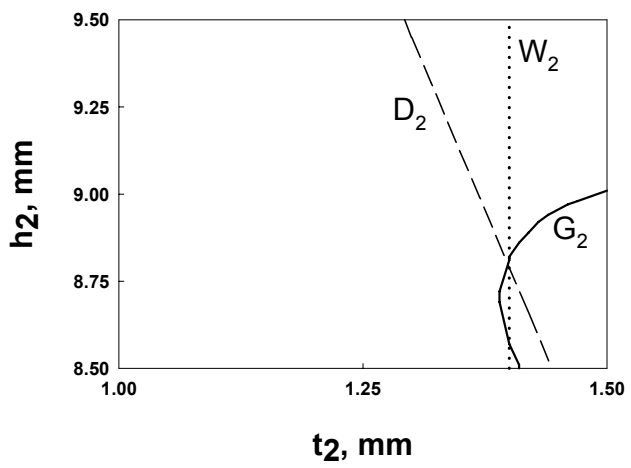

(b) $\mathrm{CSS}_{2}$

Fig. 3 Intersection of the surfaces

\section{Conclusions}

The DOE and RSM approaches have been combined to perform the simultaneous optimization of the electrical and mechanical performances of the composite smart structures consisting of antennas and integrated mechanical structures. The experiment was very simple to implement and provided a thorough understanding of the issues to be confronted to in the codesign and optimization process. The statistical analysis provided second order design equations modeling both electrical and mechanical figures of merit, then the optimization was performed simultaneously by allocating arbitrary weight factors to each one of them. By extending this approach to carefully investigate the behavior of a complex system, such as a 3D multilayer module, the designer can save a lot of time, shorten the design cycle of added functions and achieve all aspects of the design process, both electrical, mechanical, even thermal in a simple and elegant manner and with a profound understanding of how all these aspects are affecting each other.

\section{References:}

[1] A. J. Lockyer, et al., "Design and Development of a Conformal Load-Bearing Smart-Skin Antenna: Overview of the AFRL Smart Skin Structures Technology Demonstration ( $\left.{ }^{3} \mathrm{TD}\right)$ ", SPIE Smart Structures and Materials: Industrial and Commercial Application of Smart Structures Technologies, vol. 3674, pp. 410-424, 1999.

[2] A. J. Lockyer, et al., "A Qualitative Assessment of Smart Skins and Avionics/Structures Integration", SPIE Smart Structures and Materials: Smart Materials, vol. 2189, pp. 172$183,1994$.

[3] J. Tuss, et al., "Conformal Loadbearing Antenna Structure", 37th AIAA SDM Conference, pp. 836-843, 1996.

[4] Delphi Fuba Multiple Antenna Reception System, http://www.delphi.com.

[5] C.S. You, W. Hwang, "Design of Load-Bearing Antenna Structures by Embedding Technology of Microstrip Antenna in Composite Sandwich Structure", Composite Structures, Vol. 71, Issue 3-4, pp. 378-382, December 2005.

[6] N.Bushyager, D.Staiculescu, L.Martin, J.-H.Lee, N.Vasiloglou and M.M.Tentzeris, "Design and Optimization of 3D RF Modules, Microsystems and Packages Using Electromagnetic and Statistical Tools", Proc. of the 2004 IEEE-ECTC Symposium, pp.1412-1415, June 2004.

[7] D. Zenkert, "An Introduction to Sandwich Construction," EMAS Publishing, 1997

[8] D.C. Montgomery, "Design and Analysis of Experiments," New York: J. Wiley \& Sons, 1997 